\title{
Generalizing Narayana and Schröder Numbers to Higher Dimensions
}

\author{
Robert A. Sulanke \\ Boise State University \\ Boise, Idaho, USA \\ sulanke@math. boisestate.edu
}

Submitted: Dec 29, 2003; Accepted: May 15, 2004; Published: Aug 23, 2004

\begin{abstract}
Let $\mathcal{C}(d, n)$ denote the set of $d$-dimensional lattice paths using the steps $X_{1}:=$ $(1,0, \ldots, 0), X_{2}:=(0,1, \ldots, 0), \ldots, X_{d}:=(0,0, \ldots, 1)$, running from $(0,0, \ldots, 0)$ to $(n, n, \ldots, n)$, and lying in $\left\{\left(x_{1}, x_{2}, \ldots, x_{d}\right): 0 \leq x_{1} \leq x_{2} \leq \ldots \leq x_{d}\right\}$. On any path $P:=p_{1} p_{2} \ldots p_{d n} \in \mathcal{C}(d, n)$, define the statistics asc $(P):=\mid\left\{i: p_{i} p_{i+1}=X_{j} X_{\ell}, j<\right.$ $\ell\} \mid$ and $\operatorname{des}(P):=\left|\left\{i: p_{i} p_{i+1}=X_{j} X_{\ell}, j>\ell\right\}\right|$. Define the generalized Narayana number $N(d, n, k)$ to count the paths in $\mathcal{C}(d, n)$ with $\operatorname{asc}(P)=k$. We consider the derivation of a formula for $N(d, n, k)$, implicit in MacMahon's work. We examine other statistics for $N(d, n, k)$ and show that the statistics asc and des $-d+1$ are equidistributed. We use Wegschaider's algorithm, extending Sister Celine's (WilfZeilberger) method to multiple summation, to obtain recurrences for $N(3, n, k)$. We introduce the generalized large Schröder numbers $\left(2^{d-1} \sum_{k} N(d, n, k) 2^{k}\right)_{n \geq 1}$ to count constrained paths using step sets which include diagonal steps.
\end{abstract}

Key phases: Lattice paths, Catalan numbers, Narayana numbers, Schröder numbers, Sister Celine's (Wilf-Zeilberger) method

Mathematics Subject Classification: 05A15

\section{Introduction}

In $d$-dimensional coordinate space consider lattice paths that use the unit steps

$$
X_{1}:=(1,0, \ldots, 0), X_{2}:=(0,1, \ldots, 0), \ldots, X_{d}:=(0,0, \ldots, 1) .
$$

Let $\mathcal{C}(d, n)$ denote the set of lattice paths running from $(0,0, \ldots, 0)$ to $(n, n, \ldots, n)$ and lying in the region $\left\{\left(x_{1}, x_{2}, \ldots, x_{d}\right): 0 \leq x_{1} \leq x_{2} \leq \ldots \leq x_{d}\right\}$. On any path $P:=$ $p_{1} p_{2} \ldots p_{d n}$, we call any step pair $p_{i} p_{i+1}$ an ascent (respectively, a descent) if $p_{i} p_{i+1}=X_{j} X_{\ell}$ 


\begin{tabular}{|c||c|c|c|}
\hline$P \in \mathcal{C}(3,2)$ & $\operatorname{asc}(P)$ & $\operatorname{des}(P)$ & $\operatorname{hdes}(P)$ \\
\hline$Z Z Y Y X X$ & 0 & 2 & 2 \\
$Z Z Y X Y X$ & 1 & 3 & 1 \\
$Z Y Z Y X X$ & 1 & 3 & 1 \\
$Z Y Z X Y X$ & 2 & 3 & 1 \\
$Z Y X Z Y X$ & 1 & 4 & 0 \\
\hline
\end{tabular}

Table 1: For $d=3$ and $n=2$. hdes $(P)$ appears in $\S 3.2$.

for $j<\ell$ (respectively, for $j>\ell$ ). (See Remark 1.1.) To denote the statistics for the number of ascents and the number of descents, we put

$$
\begin{aligned}
\operatorname{asc}(P) & :=\mid\left\{i: p_{i} p_{i+1}=X_{j} X_{\ell} \text { for } j<\ell\right\} \mid, \\
\operatorname{des}(P) & :=\mid\left\{i: p_{i} p_{i+1}=X_{j} X_{\ell} \text { for } j>\ell\right\} \mid .
\end{aligned}
$$

For convenience when $d \leq 3$, put $X:=X_{1}, Y:=X_{2}$, and $Z:=X_{3}$. See Table 1 . For $d=2$, it is well known that, for $0 \leq k \leq n-1$,

$$
|\{P \in \mathcal{C}(2, n): \operatorname{asc}(P)=k\}|=\frac{1}{n}\left(\begin{array}{l}
n \\
k
\end{array}\right)\left(\begin{array}{c}
n \\
k+1
\end{array}\right),
$$

where the right side is called a Narayana number. See Remark 1.2.

For any dimension $d \geq 2$ and for $0 \leq k \leq(d-1)(n-1)$, we define the $d$-Narayana distribution or number, as

$$
N(d, n, k):=|\{P \in \mathcal{C}(d, n): \operatorname{asc}(P)=k\}| .
$$

Section 2 will consider establishing the formula for $N(d, n, k)$ as given in the following proposition, which is implicit in more general $q$-analogue results in MacMahon's study of plane partitions [10][11, art. 443, 451, 495][12, ch. 11]:

Proposition 1 For any dimension $d \geq 2$ and for $0 \leq k \leq(d-1)(n-1)$,

$$
N(d, n, k)=\sum_{j=0}^{k}(-1)^{k-j}\left(\begin{array}{c}
d n+1 \\
k-j
\end{array}\right) \prod_{i=0}^{d-1}\left(\begin{array}{c}
n+i+j \\
n
\end{array}\right)\left(\begin{array}{c}
n+i \\
n
\end{array}\right)^{-1} .
$$

For $d \geq 2$ and $n \geq 1$, we define the $n$-th $d$-Narayana polynomial to be

$$
N_{d, n}(t):=\sum_{k=0}^{(d-1)(n-1)} N(d, n, k) t^{k}
$$

with $N_{d, 0}(t):=1$. The sequence $\left(N_{d, n}(1)\right)_{n \geq 0}$ has been called the $d$-dimensional Catalan numbers. For $n \geq 0$, we have the known formula (See [11, art. 93-103][28]; sequence A005789 in [17].):

$$
N_{d, n}(1)=(d n) ! \prod_{i=0}^{d-1} \frac{i !}{(n+i) !}
$$


which we will reconsider for $d=3$ in Proposition 8. For arbitrary $t$ and for $d=3$,

$$
\begin{gathered}
N_{3,0}(t)=1 \\
N_{3,1}(t)=1 \\
N_{3,2}(t)=1+3 t+t^{2} \\
N_{3,3}(t)=1+10 t+20 t^{2}+10 t^{3}+t^{4} \\
N_{3,5}(t)=1+40 t+400 t^{2}+1456 t^{3}+2212 t^{4}+1456 t^{5}+400 t^{6}+40 t^{7}+t^{8}
\end{gathered}
$$

In Section 3 we will examine the statistic des and other statistics which are also distributed by the $d$-Narayana distribution. When $d=2$, since the locations of the descents and the ascents alternate on any path $P \in \mathcal{C}(2, n)$, certainly $\operatorname{des}(P)=\operatorname{asc}(P)+1$. However, when $d=3$, a relationship between these two statistics is not apparent as Table 1 should show. We will prove bijectively that

Proposition 2 For $d \geq 2$ and $n \geq 1$, the statistics asc and des $-d+1$ are equally distributed on $\mathcal{C}(d, n)$. Hence,

$$
\sum_{P \in \mathcal{C}(d, n)} t^{\operatorname{asc}(P)}=\sum_{P \in \mathcal{C}(d, n)} t^{\operatorname{des}(P)-d+1}=N_{d, n}(t) .
$$

In Section 4 we will use an algorithm of Wegschaider [26], which extends the WilfZeilberger multivariate generalization of Sister Celine's method, to obtain some recurrences for $N_{3, n}(t)$ and for $N(d, n, k)$.

In Section 5 we will introduce a $d$-dimensional analogue of the large Schröder numbers as the sequence $\left(2^{d-1} N_{d, n}(2)\right)_{n \geq 1}$. It will follow from Proposition 2 that this sequence counts paths running from $(0,0, \ldots, 0)$ to $(n, n, \ldots, n)$, lying in $\left\{\left(x_{1}, x_{2}, \ldots, x_{n}\right): 0 \leq\right.$ $\left.x_{1} \leq x_{2} \leq \ldots \leq x_{n}\right\}$, and using positive steps of the form $\left(\xi_{1}, \xi_{2}, \ldots, \xi_{n}\right)$ where $\xi_{i} \in$ $\{0,1\}$. It will also follow that $2^{d+n-2} N_{d, n}(2)$ counts the paths running from $(0,0, \ldots, 0)$ to $(n, n, \ldots, n)$, lying in $\left\{\left(x_{1}, x_{2}, \ldots, x_{n}\right): 0 \leq x_{1} \leq x_{2} \leq \ldots \leq x_{n}\right\}$, and using positive steps of the form $\left(\xi_{1}, \xi_{2}, \ldots, \xi_{n}\right)$ where $\xi_{i}$ is a nonnegative integer.

\section{Remarks:}

1.1. The paths of $\mathcal{C}(d, n)$ are also called ballot paths for $d$ candidates, or lattice permutations as in MacMahon [11]. If the condition constraining that paths of $\mathcal{C}(d, n)$ is replaced by $0 \leq x_{d} \leq x_{d-1} \leq \ldots \leq x_{2} \leq x_{1}$, then our results in terms of ascents become ones for descents, and vice versa.

1.2. The right side of (1) is named for Narayana who introduced the formula in 1955 [13]. However, this formula is immediately a special case of an earlier formula of MacMahon [11, art. 495, 5th formula]. Proposition 1 shows that the right side of (1) indeed agrees with (3) for $d=2$. See $[23,24]$ for studies of $N(2, n, k)$.

In 1910 MacMahon [10, 11] introduced the sub-lattice function of order $k$, which is a $q$-analogue of $N(d, n, k)$. This might be the earliest appearance of the $d$-dimensional Narayana numbers. 
1.3. One can express $N(d, n, k)$ as the number of rectangular standard Young tableaux with $d$ rows and $n$ columns having $k$ occurrences of an integer $i$ appearing in a lower row than that of $i+1$. It is the terminology of lattice paths, however, that allows results admitting diagonal steps and hence the generalization of the Schröder numbers to higher dimensions.

1.4. In [25] the author studies counting $\mathcal{C}(3, n)$ with respect to the statistic des and obtains a formula for 3-Narayana numbers which is quite different from the formula of (3).

\section{Counting paths with respect to ascents}

We now indicate how formula (3), producing the $d$-Narayana numbers, is a consequence of Stanley's theory of $P$-partitions [18, 20], even though, (3) is implicit in MacMahon's work. We do so to give perspective and to facilitate obtaining another statistic having the $d$-Narayana distribution in $\S 3.2$. We remark that, while Stanley's theory extends results of MacMahon for plane partitions, notational differences cause their specializations to (3) to be different. We will also consider the reciprocity of the Narayana polynomials.

Some notation is required with details appearing in [20]. For any positive integer $n$, let $[n]:=\{1,2, \ldots, n\}$ and let $\mathbf{n}$ denote the chain $1<2<\cdots<n$. For any finite partially ordered set (poset) $\mathbf{P}$, with $p:=|\mathbf{P}|$, a linear extension of $\mathbf{P}$ is an order preserving bijection $\sigma: \mathbf{P} \rightarrow \mathbf{p}$. We remark that a specified linear extension of $\mathbf{P}$ is a labeling of the set $\mathbf{P}$, which corresponds to $\mathbf{P}$ being a natural partial order on $[p]$, as in [20]. For a specified linear extension $\omega: \mathbf{P} \rightarrow \mathbf{p}$,

$$
\mathcal{L}(\mathbf{P}, \omega):=\left\{\omega \circ \sigma^{-1}: \sigma \text { is a linear extension of } \mathbf{P}\right\},
$$

a subset of permutations on $[p]$, called the Jordan-Hölder set. In any permutation $\tau:=$ $\tau_{1} \ldots \tau_{n}$ of $[n], \tau_{i}$ is called a descent of $\tau$ if $\tau_{i}>\tau_{i+1} ; \operatorname{DES}(\tau)$ will denote the number of descents on $\tau$.

Let $M(d, n, k)$ denote the number of plane partitions having at most $d$ rows, at most $n$ columns, and part size at most $m$. It is easily seen that $M(d, n, m)$ is equal to the order polynomial $\Omega(\mathbf{d} \times \mathbf{n}, m+1)$, which is defined as the number of order-preserving maps from the direct product poset $\mathbf{d} \times \mathbf{n}$ to $[m+1]$.

From a fundamental property of order polynomials, specifically from [20, Theorem 5.4.14],

$$
\sum_{m \geq 0} \Omega(\mathbf{P}, m) \lambda^{m}=(1-\lambda)^{-p-1} \sum_{\pi \in \mathcal{L}(\mathbf{P}, \omega)} \lambda^{1+\operatorname{DES}(\pi)},
$$

we obtain a convolution for our purposes:

Proposition 3 For positive integers $d, n$, and $m$, and for specified linear extension $\omega$,

$$
M(d, n, m)=\sum_{k \geq 0}\left(\begin{array}{c}
d n+m-k \\
d n
\end{array}\right)|\{\tau \in \mathcal{L}(\mathbf{d} \times \mathbf{n}, \omega): \operatorname{DES}(\tau)=k\}| .
$$



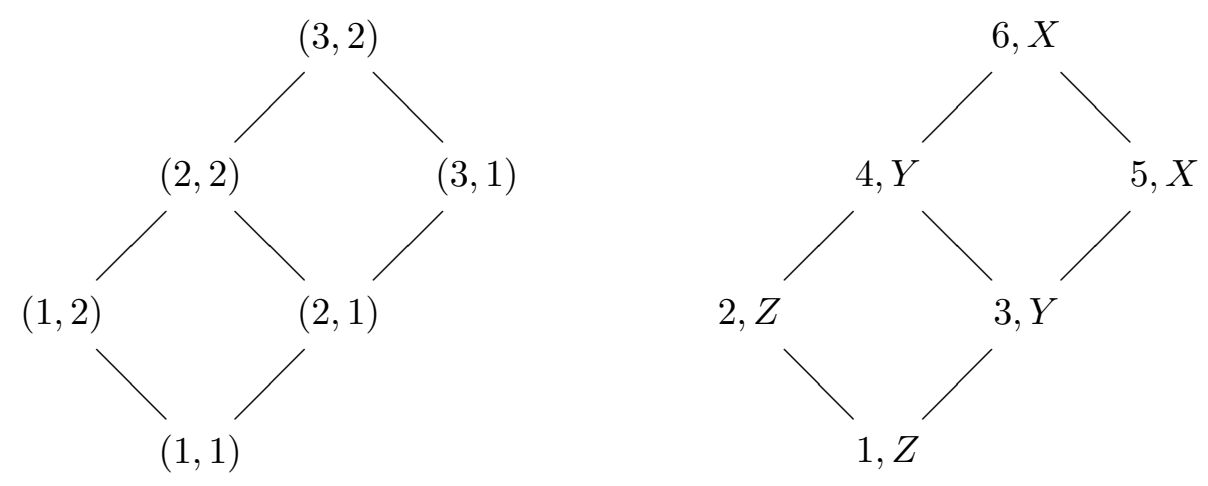

Figure 1: The poset $\mathbf{d} \times \mathbf{n}=\mathbf{3} \times \mathbf{2}$ and its labeled version.

\begin{tabular}{|c|c||c|c|}
\hline permutation $\tau \in \mathcal{L}$ & DEs $(\tau)$ & path $P \in C(3,2)$ & $\operatorname{asc}(P)$ \\
\hline 123456 & 0 & $Z Z Y Y X X$ & 0 \\
123546 & 1 & $Z Z Y X Y X$ & 1 \\
132456 & 1 & $Z Y Z Y X X$ & 1 \\
135246 & 1 & $Z Y X Z Y X$ & 1 \\
132546 & 2 & $Z Y Z X Y X$ & 2 \\
\hline
\end{tabular}

Table 2:

To apply (4) in terms of $N(d, n, k)$ we will assign two labels to each point of $\mathbf{d} \times \mathbf{n}$. For the first labeling we specify the linear extension

$$
\omega: \mathbf{d} \times \mathbf{n} \rightarrow[d n]: \omega(i, j)=j+n(i-1) .
$$

For the second labeling we label $\mathbf{d} \times \mathbf{n}$ so that each $(i, j)$ receives the step $X_{d-i+1}$. These two labelings yield a simple bijection mapping each permutation $\tau$ of $\mathcal{L}(\mathbf{d} \times \mathbf{n}, \omega)$ with $\operatorname{DES}(\tau)=k$ to a path $P$ of $N(d, n, k)$ with $\operatorname{asc}(P)=k$. This bijection is evident from the example of Figure 1 and the corresponding Table 2.

Concerning the left side of (4), MacMahon [11, Art. 495] (See Remark 2.1.) was the first to find a formula for $M(d, n, m)$, which we write as

$$
M(d, n, m)=\prod_{i=0}^{d-1}\left(\begin{array}{c}
m+n+i \\
n
\end{array}\right)\left(\begin{array}{c}
n+i \\
n
\end{array}\right)^{-1} .
$$

Hence, Proposition 3 yields

Proposition 4 For $d \geq 2, m \geq 1$, and $n \geq 1$,

$$
\prod_{i=0}^{d-1}\left(\begin{array}{c}
m+n+i \\
n
\end{array}\right)\left(\begin{array}{c}
n+i \\
n
\end{array}\right)^{-1}=\sum_{k \geq 0}\left(\begin{array}{c}
d n+m-k \\
d n
\end{array}\right) N(d, n, k) .
$$


This in turn yields Proposition 1 by a simple inversion. Next, as a consequence of (6), we have

Corollary 1 For $d \geq 2$ and $n \geq 1, N_{d, n}(t)$ is a reciprocal polynomial of degree $(d-1)(n-$ $1)$. That is, for each $n$, the sequence of coefficients of $N_{d, n}(t)$ is symmetric.

Proof. This proof is similar to that of [10, art. 29]; the argument in [11, art. 449] seems incomplete. A proof can also be based on a result in [18, sect. 18] or [20, Cor. 4.5.17].

We observe that the degree of $N_{d, n}(t)$ cannot exceed $(d n-1)-(d-2)-n=(d-1)(n-1)$ since there are $d n-1$ step pairs on any path, since each of the final occurrences of the steps $X_{2}, \ldots, X_{d-1}$ on a path of $\mathcal{C}(d, n)$ cannot immediately precede an ascent, and since every $X_{d}$ step cannot immediately precede an ascent.

Recall that for real $r$, the binomial coefficient is defined so $\left(\begin{array}{l}r \\ k\end{array}\right):=\prod_{j=0}^{k-1}(r-j) / k$ ! if $k$ is a positive integer and so $\left(\begin{array}{l}r \\ 0\end{array}\right):=1$. Since the equation (6) is a polynomial equation in $m$ which is valid for all positive integer values of $m$, it is valid for all real $m$. Indeed, replacing $m$ by $-d-m-n$ in (6) yields

$$
\sum_{k \geq 0}\left(\begin{array}{c}
d n-d-m-n-k \\
d n
\end{array}\right) N(d, n, k)=\prod_{j=0}^{d-1}\left(\begin{array}{c}
-d-m+j \\
n
\end{array}\right)\left(\begin{array}{c}
n+j \\
n
\end{array}\right)^{-1} .
$$

Upon applying the well-known identity, $\left(\begin{array}{l}r \\ k\end{array}\right)=(-1)^{k}\left(\begin{array}{c}k-r-1 \\ k\end{array}\right)$, to each factor of the numerator of the right side and then commuting the factors, we find

$$
\prod_{j=0}^{d-1}\left(\begin{array}{c}
-d-m+j \\
n
\end{array}\right)\left(\begin{array}{c}
n+j \\
n
\end{array}\right)^{-1}=(-1)^{d n} \prod_{j=0}^{d-1}\left(\begin{array}{c}
m+n+j \\
n
\end{array}\right)\left(\begin{array}{c}
n+j \\
n
\end{array}\right)^{-1}
$$

Hence,

$$
\sum_{k \geq 0}\left(\begin{array}{c}
(d-1)(n-1)-m-1-k \\
d n
\end{array}\right) N(d, n, k)=(-1)^{d n} \sum_{k \geq 0}\left(\begin{array}{c}
d n+m-k \\
d n
\end{array}\right) N(d, n, k) .
$$

Recalling that the degree of $N_{d, n}(t)$ cannot exceed $(d-1)(n-1)$ and setting $m=0$, we find that the only nonzero terms in (7) correspond to $k=(d-1)(n-1)$ on the left side and to $k=0$ on the right side. Hence, $N(d, n,(d-1)(n-1))=N(d, n, 0)$. Next, repeatedly setting $m=1,2, \ldots$ and solving yield $N(d, n,(d-1)(n-1)-k)=N(d, n, k)$ for $0<k<(d-1)(n-1)$.

\section{Remarks:}

2.1. An inductive proof of (5) due to Carlitz appears in $[12, \S 11.2]$. Proofs of (5) using the Gessel-Viennot method appear in [3, Ch. 3],[7]; those concerning Schur functions appear in [3, Ch. 4], [21, §7.21]. A neat alternative to formula (5) appears at the end of $[21, \S 7.21]$.

2.2. Let $\mathcal{N} \mathcal{I}(d, n, m)$ denote the set of $d$-tuples of nonintersecting planar lattice paths, $\left(P_{1}, \ldots, P_{j}, \ldots, P_{d}\right)$, where path $P_{j}$ uses the steps $(1,0)$ and $(0,1)$ and runs from $(-d+$ 
$j, d-j)$ to $(m-d+j, n+d-j)$, for $1 \leq j \leq d$. There is an easily observed bijection between $\mathcal{N} \mathcal{I}(d, n, m)$ and the set of bounded plane partitions counted by $M(d, n, m)$ (see e.g., [3, Ch. 3],[7]). Thus the Proposition 6 is equivalent to the following which relates the number of $d$-tuples of nonintersecting paths to the number of restricted $d$-dimensional paths with respect to ascents:

$$
|\mathcal{N} \mathcal{I}(m, n, d)|=\sum_{k \geq 0}\left(\begin{array}{c}
d n+m-k \\
d n
\end{array}\right) N(d, n, k) .
$$

Kreweras [8] has given a more general result which is in terms of skew tableaux.

2.3. For $d=2$, (5) easily simplifies to $\frac{1}{m+n+1}\left(\begin{array}{c}m+n+1 \\ m\end{array}\right)\left(\begin{array}{c}m+n+1 \\ m+1\end{array}\right)$. Thus, Proposition 4 yields the following identity for the common Narayana numbers:

$$
N(2, n+m+1, m)=\sum_{j=0}^{m}\left(\begin{array}{c}
2 n+j \\
j
\end{array}\right) N(2, n, n-j) .
$$

2.4. Our interest in knowing a formula such as (3) was motivated by a study of Kreweras and Niederhausen [9], which concerned 3-dimensional paths constrained by $\max \{x, y\} \leq z$. Recently Brändén [2] used an approach similar to that of this section in studying statistics distributed by a $q$-analogue of the Narayana distribution for $d=2$.

\section{Other statistics having the $d$-Narayana distribution}

\subsection{A bijective proof that asc and des $-d+1$ are equidistributed.}

For $n \geq 1$ and $d \geq 2$, we will consider statistics on $\mathcal{C}(d, n)$, each of which is expressed (or encoded) in terms of a $d$ by $d$ 0-1 matrix $M$. Here $(M)_{j \ell}$ denotes the entry in row $j$ and column $\ell$ of $M$, while $M_{i j}$ denotes a specific matrix identified by the subscripts. Let $\Theta_{M}$ denote a statistic on $\mathcal{C}(d, n)$ defined so that, for each path $P:=p_{1} p_{2} \ldots p_{d n}$,

$$
\Theta_{M}(P):=\sum_{j=1}^{d} \sum_{\ell=1}^{d}(M)_{j \ell}\left|\left\{i: p_{i} p_{i+1}=X_{j} X_{\ell}, 1 \leq i<d n\right\}\right| .
$$

Define the matrices $M_{A}$ and $M_{D}$ so

$$
\begin{aligned}
& \left(M_{A}\right)_{j \ell}:=1 \text { if } j<\ell, \text { and }=0 \text { if otherwise, } \\
& \left(M_{D}\right)_{j \ell}:=1 \text { if } j>\ell, \text { and }=0 \text { if otherwise. }
\end{aligned}
$$

Hence, $\operatorname{asc}(P)=\Theta_{M_{A}}(P)$ and $\operatorname{des}(P)=\Theta_{M_{D}}(P)$.

Throughout this section we will use a detailed treatment of the case for $d=4$ to afford clarity to the general case. For example, for $d=4$, the statistic asc corresponds to the matrix

$$
M_{A}:=V M_{33}=\left[\begin{array}{llll}
0 & 1 & 1 & 1 \\
0 & 0 & 1 & 1 \\
0 & 0 & 0 & 1 \\
0 & 0 & 0 & 0
\end{array}\right],
$$




$$
\begin{aligned}
& {\left[\begin{array}{llll}
0 & 0 & 0 & 0 \\
1 & 0 & 0 & 0 \\
1 & 1 & 0 & 0 \\
1 & 1 & 1 & 0
\end{array}\right] \stackrel{H}{\longrightarrow}\left[\begin{array}{llll}
0 & 0 & 0 & 0 \\
0 & 1 & 1 & 1 \\
0 & 0 & 1 & 1 \\
0 & 0 & 0 & 1
\end{array}\right] \stackrel{V}{\longrightarrow}\left[\begin{array}{llll}
0 & 1 & 1 & 1 \\
0 & 0 & 0 & 0 \\
0 & 1 & 0 & 0 \\
0 & 1 & 1 & 0
\end{array}\right] \stackrel{H}{\longrightarrow}\left[\begin{array}{llll}
1 & 0 & 0 & 0 \\
0 & 0 & 0 & 0 \\
1 & 0 & 1 & 1 \\
1 & 0 & 0 & 1
\end{array}\right] \stackrel{T_{2}}{\longrightarrow}} \\
& {\left[\begin{array}{llll}
1 & 0 & 1 & 1 \\
0 & 0 & 0 & 0 \\
0 & 0 & 1 & 1 \\
0 & 0 & 0 & 1
\end{array}\right] \stackrel{V}{\longrightarrow}\left[\begin{array}{llll}
0 & 0 & 0 & 0 \\
1 & 0 & 1 & 1 \\
1 & 0 & 0 & 0 \\
1 & 0 & 1 & 0
\end{array}\right] \stackrel{H}{\longrightarrow}\left[\begin{array}{llll}
0 & 0 & 0 & 0 \\
0 & 1 & 0 & 0 \\
0 & 1 & 1 & 1 \\
0 & 1 & 0 & 1
\end{array}\right] \stackrel{V}{\longrightarrow}\left[\begin{array}{llll}
0 & 1 & 1 & 1 \\
0 & 0 & 1 & 1 \\
0 & 0 & 0 & 0 \\
0 & 0 & 1 & 0
\end{array}\right] \stackrel{H}{\longrightarrow}\left[\begin{array}{llll}
1 & 0 & 0 & 0 \\
1 & 1 & 0 & 0 \\
0 & 0 & 0 & 0 \\
1 & 1 & 0 & 1
\end{array}\right] \stackrel{T_{3}}{\longrightarrow}}
\end{aligned}
$$

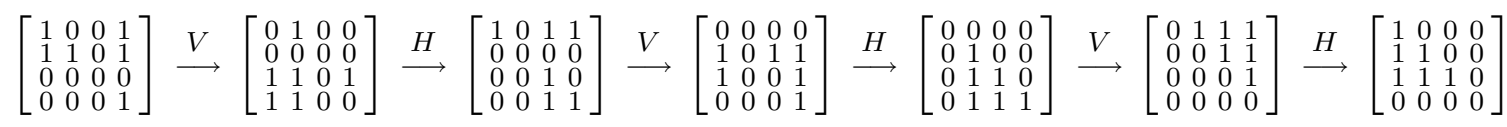

$$
\begin{aligned}
& M_{D} \quad M_{11} \quad V M_{11} \quad M_{12} \\
& \begin{array}{lllll}
M_{21} & V M_{21} & M_{22} & M_{22}
\end{array} \\
& \begin{array}{lllllll}
M_{31} & V M_{31} & M_{32} & V M_{32} & M_{33} & V M_{33} & M_{34}
\end{array}
\end{aligned}
$$

Figure 2: The top 3 lines give the schema for the proof of Proposition 2. The bottom 3 lines relate the notation. The definition of $T_{i}$ appears after Lemma 4 .

since

$$
\operatorname{asc}(P)=\mid\left\{i: p_{i} p_{i+1}=X_{j} X_{\ell}, \text { for } j<\ell\right\} \mid \text {. }
$$

(We explain the " $V$ " and the "33" momentarily.) Similarly, the statistic des corresponds to the matrix

$$
M_{D}:=H M_{11}=\left[\begin{array}{llll}
0 & 0 & 0 & 0 \\
1 & 0 & 0 & 0 \\
1 & 1 & 0 & 0 \\
1 & 1 & 1 & 0
\end{array}\right] .
$$

For each matrix $M$ under consideration, we define the horizontal complement, $H M$, and the vertical complement, $V M$, to be matrices defined so

$$
\begin{aligned}
& (H M)_{j \ell}:= \begin{cases}0 & \text { if } j \text { is a zero row of } M \\
1-(M)_{j \ell} & \text { if otherwise, }\end{cases} \\
& (V M)_{j \ell}:= \begin{cases}0 & \text { if } \ell \text { is a zero column of } M \\
1-(M)_{j \ell} & \text { if otherwise. }\end{cases}
\end{aligned}
$$

(E.g., see the top of Figure 2; see also $M_{73}, V M_{73}$, and $M_{74}$ in Figure 3.)

Lemma 1 For any d by d matrix $M$ having exactly one row and one column of 0 's,

$$
\begin{aligned}
\Theta_{M}(P)+\Theta_{H M}(P) & = \begin{cases}(d-1) n & \text { if the first row of } M \text { is a zero row } \\
(d-1) n-1 & \text { if otherwise, }\end{cases} \\
\Theta_{M}(P)+\Theta_{V M}(P) & = \begin{cases}(d-1) n & \text { if the last column of } M \text { is a zero column } \\
(d-1) n-1 & \text { if otherwise. }\end{cases}
\end{aligned}
$$




$$
\begin{aligned}
& M_{11} V M_{11} \quad M_{12} \\
& M_{21} \quad V M_{21} \quad M_{22} \quad V M_{22} \quad M_{23} \\
& \begin{array}{lllllll}
M_{31} & V M_{31} & M_{32} & V M_{32} & M_{33} & V M_{33} & M_{34}
\end{array} \\
& \text {... } \\
& \begin{array}{lllllllll}
M_{i 1} & V M_{i 1} & M_{i 2} & V M_{i 2} & \ldots & V M_{i, i-1} & M_{i i} & V M_{i i} & M_{i, i+1}
\end{array} \\
& M_{d-1,1} V M_{d-1,1} \quad M_{d-1,2} \quad V M_{d-1,2} \quad \ldots V M_{d-1, d-2} \quad M_{d-1, d-1} \quad V M_{d-1, d-1} \quad M_{d-1, d} .
\end{aligned}
$$

Table 3: The trapezoidal array of matrices.

Table 4: The zero intersections for $d=4$.

Proof. We note that each path begins with $X_{d}$, ends with $X_{1}$, and has a total of $d n-1$ consecutive step pairs. If row 1 of $M$ is a zero row, then the $n-1$ non-final $X_{1}$ steps, all of which immediately precede some other step on $P$, do not contribute to $\Theta_{M}(P)+\Theta_{H M}(P)$. Hence, $\Theta_{M}(P)+\Theta_{H M}(P)=(d n-1)-(n-1)$. If row 2 of $M$ is a zero row, then only the $n X_{2}$ steps, which must immediately precede some other step on $P$, do not contribute to $\Theta_{M}(P)+\Theta_{H M}(P)=(d n-1)-n$. Similarly, the other instances of the lemma are valid.

We now define the trapezoidal array of matrices appearing in Table 3 (and illustrated for $d=4$ in Figure 2). For $1 \leq i \leq d-1$, we define $M_{i 1}$ so that

$$
\left(M_{i 1}\right)_{j \ell}:= \begin{cases}1 & \text { if } \ell \leq j<i \text { or } j<i<\ell \text { or } i<j \leq \ell \\ 0 & \text { if otherwise. }\end{cases}
$$

Moreover, for $1 \leq j \leq i \leq d-1$, define $M_{i, j+1}:=H V M_{i, j}$. (E.g., see Figures 2 and 3.)

Given each matrix in the trapezoidal array, it is useful to determine the indices of the intersection of its zero row and zero column, called its zero intersection. One can check that the array of Table 4 gives the zero intersections corresponding to the the trapezoidal array of matrices for $d=4$. More generally we state a lemma.

Lemma 2 Let $d \geq 3$. For $1 \leq j<i \leq d-1$, the zero intersection of $M_{i, j}$ has indices $(i+1-j, i+1-j)$ and the zero intersection of $V M_{i, j}$ has indices $(i-j, i+1-j)$. The zero intersection of $M_{i, i}$ has indices $(1,1)$, the zero intersection of $V M_{i, i}$ has indices $(i+1,1)$, and the zero intersection of $M_{i, i+1}$ has indices $(i+1, i+1)$.

Proof. Without introducing awkward notation, one can check the validity of this lemma by working through the examples of Figures 2 and 3 which are sufficiently general 


$$
\begin{aligned}
& M_{67}=\left[\begin{array}{llllllllll}
1 & 0 & 0 & 0 & 0 & 0 & \mathbf{0} & 0 & 0 & 0 \\
1 & 1 & 0 & 0 & 0 & 0 & \mathbf{0} & 0 & 0 & 0 \\
1 & 1 & 1 & 0 & 0 & 0 & \mathbf{0} & 0 & 0 & 0 \\
1 & 1 & 1 & 1 & 0 & 0 & \mathbf{0} & 0 & 0 & 0 \\
1 & 1 & 1 & 1 & 1 & 0 & \mathbf{0} & 0 & 0 & 0 \\
1 & 1 & 1 & 1 & 1 & 1 & \mathbf{0} & 0 & 0 & 0 \\
\mathbf{0} & \mathbf{0} & \mathbf{0} & \mathbf{0} & \mathbf{0} & \mathbf{0} & \mathbf{0} & \mathbf{0} & \mathbf{0} & \mathbf{0} \\
\mathbf{1} & \mathbf{1} & \mathbf{1} & \mathbf{1} & \mathbf{1} & \mathbf{1} & \mathbf{0} & 1 & 1 & 1 \\
\mathbf{1} & \mathbf{1} & \mathbf{1} & \mathbf{1} & \mathbf{1} & \mathbf{1} & \mathbf{0} & 0 & 1 & 1 \\
\mathbf{1} & \mathbf{1} & \mathbf{1} & \mathbf{1} & \mathbf{1} & \mathbf{1} & \mathbf{0} & 0 & 0 & 1
\end{array}\right] \quad \longrightarrow \quad T_{7} \quad \longrightarrow \quad M_{71}=\left[\begin{array}{llllllllll}
1 & 0 & 0 & 0 & 0 & 0 & \mathbf{0} & \mathbf{1} & \mathbf{1} & \mathbf{1} \\
1 & 1 & 0 & 0 & 0 & 0 & \mathbf{0} & \mathbf{1} & \mathbf{1} & \mathbf{1} \\
1 & 1 & 1 & 0 & 0 & 0 & \mathbf{0} & \mathbf{1} & \mathbf{1} & \mathbf{1} \\
1 & 1 & 1 & 1 & 0 & 0 & \mathbf{0} & \mathbf{1} & \mathbf{1} & \mathbf{1} \\
1 & 1 & 1 & 1 & 1 & 0 & \mathbf{0} & \mathbf{1} & \mathbf{1} & \mathbf{1} \\
1 & 1 & 1 & 1 & 1 & 1 & \mathbf{0} & \mathbf{1} & \mathbf{1} & \mathbf{1} \\
\mathbf{0} & \mathbf{0} & \mathbf{0} & \mathbf{0} & \mathbf{0} & \mathbf{0} & \mathbf{0} & \mathbf{0} & \mathbf{0} & \mathbf{0} \\
0 & 0 & 0 & 0 & 0 & 0 & \mathbf{0} & 1 & 1 & 1 \\
0 & 0 & 0 & 0 & 0 & 0 & \mathbf{0} & 0 & 1 & 1 \\
0 & 0 & 0 & 0 & 0 & 0 & \mathbf{0} & 0 & 0 & 1
\end{array}\right] \quad(H V)^{2}
\end{aligned}
$$

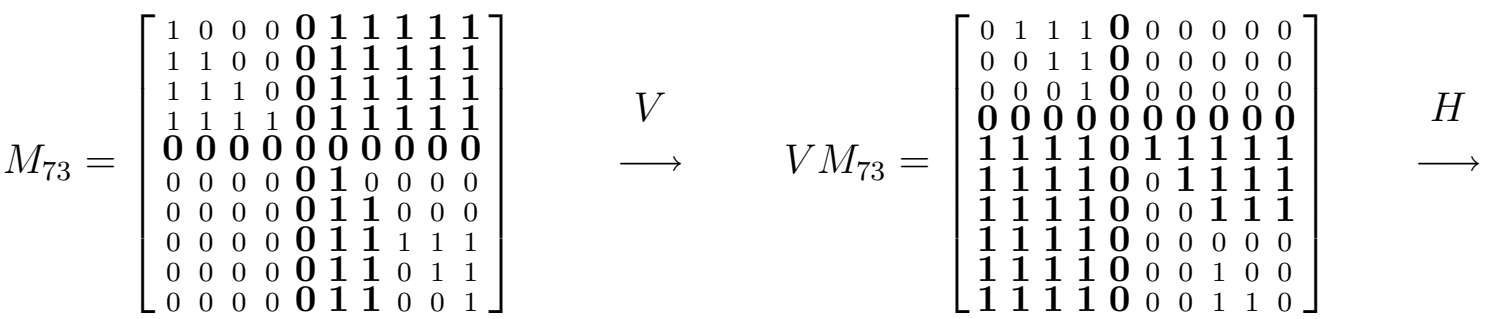

$$
\begin{aligned}
& M_{74}=\left[\begin{array}{llllllllll}
1 & 0 & 0 & \mathbf{0} & \mathbf{1} & \mathbf{1} & \mathbf{1} & \mathbf{1} & \mathbf{1} & \mathbf{1} \\
1 & 1 & 0 & \mathbf{0} & \mathbf{1} & \mathbf{1} & \mathbf{1} & \mathbf{1} & \mathbf{1} & \mathbf{1} \\
1 & 1 & 1 & \mathbf{0} & \mathbf{1} & \mathbf{1} & \mathbf{1} & \mathbf{1} & \mathbf{1} & \mathbf{1} \\
\mathbf{0} & \mathbf{0} & \mathbf{0} & \mathbf{0} & \mathbf{0} & \mathbf{0} & \mathbf{0} & \mathbf{0} & \mathbf{0} & \mathbf{0} \\
0 & 0 & 0 & \mathbf{0} & \mathbf{1} & 0 & 0 & 0 & 0 & 0 \\
0 & 0 & 0 & \mathbf{0} & \mathbf{1} & \mathbf{1} & 0 & 0 & 0 & 0 \\
0 & 0 & 0 & \mathbf{0} & \mathbf{1} & \mathbf{1} & \mathbf{1} & 0 & 0 & 0 \\
0 & 0 & 0 & \mathbf{0} & \mathbf{1} & \mathbf{1} & \mathbf{1} & 1 & 1 & 1 \\
0 & 0 & 0 & \mathbf{0} & \mathbf{1} & \mathbf{1} & \mathbf{1} & 0 & 1 & 1 \\
0 & 0 & 0 & \mathbf{0} & \mathbf{1} & \mathbf{1} & \mathbf{1} & 0 & 0 & 1
\end{array}\right] \quad V(H V)^{2} \quad \longrightarrow \quad W \quad \longrightarrow\left[\begin{array}{llllllllll}
\mathbf{0} & \mathbf{0} & \mathbf{0} & \mathbf{0} & \mathbf{0} & \mathbf{0} & \mathbf{0} & \mathbf{0} & \mathbf{0} & \mathbf{0} \\
\mathbf{1} & \mathbf{0} & \mathbf{1} & \mathbf{1} & \mathbf{1} & \mathbf{1} & \mathbf{1} & \mathbf{1} & \mathbf{1} & \mathbf{1} \\
\mathbf{1} & \mathbf{0} & 0 & \mathbf{1} & \mathbf{1} & \mathbf{1} & \mathbf{1} & \mathbf{1} & \mathbf{1} & \mathbf{1} \\
\mathbf{1} & \mathbf{0} & 0 & 0 & \mathbf{1} & \mathbf{1} & \mathbf{1} & \mathbf{1} & \mathbf{1} & \mathbf{1} \\
\mathbf{1} & \mathbf{0} & 0 & 0 & 0 & \mathbf{1} & \mathbf{1} & \mathbf{1} & \mathbf{1} & \mathbf{1} \\
\mathbf{1} & \mathbf{0} & 0 & 0 & 0 & 0 & \mathbf{1} & \mathbf{1} & \mathbf{1} & \mathbf{1} \\
\mathbf{1} & \mathbf{0} & 0 & 0 & 0 & 0 & 0 & \mathbf{1} & \mathbf{1} & \mathbf{1} \\
\mathbf{1} & \mathbf{0} & 0 & 0 & 0 & 0 & 0 & 0 & 0 & 0 \\
\mathbf{1} & \mathbf{0} & 0 & 0 & 0 & 0 & 0 & 1 & 0 & 0 \\
\mathbf{1} & \mathbf{0} & 0 & 0 & 0 & 0 & 0 & 1 & 1 & 0
\end{array}\right]
\end{aligned}
$$

Figure 3: This illustrates the action of $T_{7}, H$, and $V$.

to explain the actions of $V$ and $H$ on $M_{i j}$. (Momentarily, ignore the actions of $T_{2}, T_{3}$ and $T_{7}$.) Starting with the second matrix, $M_{71}$, at each stage one should pay particular attention to how the submatrix lying below the zero row and to the right of the zero column is transformed.

Lemma 3 For $1 \leq j \leq i \leq d-1$,

$$
\begin{aligned}
\Theta_{M_{i j}}(P)+\Theta_{V M_{i j}}(P) & =(d-1) n-1 \\
\Theta_{V M_{i j}}(P)+\Theta_{M_{i, j+1}}(P) & = \begin{cases}(d-1) n & \text { if } j=i-1 \\
(d-1) n-1 & \text { if otherwise }\end{cases}
\end{aligned}
$$

Consequently, for $2 \leq i \leq d-1$,

$$
\Theta_{M_{i 1}}(P)+\Theta_{M_{i, i+1}}(P)= \begin{cases}0 & \text { if } i=1 \\ 1 & \text { if } 2 \leq i \leq d-1\end{cases}
$$

Proof. Use Lemmas 1 and 2. The second part relies on telescopic cancellation. 
Lemma 4 For any $d \geq 2$, since $H M_{D}=M_{11}$ and $M_{A}=H M_{d-1, d}$,

$$
\begin{aligned}
\operatorname{des}(P)+\Theta_{M_{11}}(P) & =(d-1) n \\
\operatorname{asc}(P)+\Theta_{M_{d-1, d}}(P) & =(d-1) n-1
\end{aligned}
$$

For any $d$ by $d$ matrix $M$ and for $2 \leq i \leq d-1$, we define a "restricted transpose", denoted by $T_{i} M$, so that

$$
\left(T_{i} M\right)_{j \ell}:= \begin{cases}(M)_{\ell j} & \text { if } \ell<i<j \text { or } j<i<\ell \\ (M)_{j \ell} & \text { if otherwise. }\end{cases}
$$

Observe that $T_{i} M_{i-1, i}=M_{i 1}$. (E.g., see the top row of Figure 3.)

Moreover, for $2 \leq i \leq d-1$, we define a bijection

$$
\beta_{i}: \mathcal{C}(d, n) \rightarrow \mathcal{C}(d, n)
$$

as follows: For any $P \in \mathcal{C}(d, n)$, break $P$ into maximal blocks which either contain only $X_{i}$ steps or contain no $X_{i}$ step. In each block of the second type, we exchange the initial maximal subblock (perhaps empty) of steps belonging to $\left\{X_{1} \ldots X_{i-1}\right\}$ with the final maximal subblock (perhaps empty) of steps belonging to $\left\{X_{1} \ldots X_{i-1}\right\}$. The resulting path is denoted as $\beta_{i}(P)$.

Example. For $d=4$, one can check that, if

$$
P=X_{4} X_{3} X_{2} X_{4} X_{3} X_{1} X_{4} X_{4} X_{2} X_{1} X_{3} X_{2} X_{1} X_{3} X_{2} X_{1}
$$

then

$$
\beta_{3}(P)=X_{4} X_{3} X_{4} X_{2} X_{3} X_{2} X_{1} X_{4} X_{4} X_{1} X_{3} X_{2} X_{1} X_{3} X_{2} X_{1} .
$$

One can also check that $\Theta_{M_{23}}(P)=\Theta_{T_{2} M_{23}}\left(\beta_{3}(P)\right)=4$ where

$$
M_{23}:=\left[\begin{array}{llll}
1 & 0 & 0 & 0 \\
1 & 1 & 0 & 0 \\
0 & 0 & 0 & 0 \\
1 & 1 & 0 & 1
\end{array}\right] \quad \text { and } \quad T_{3} M_{23}=M_{31}:=\left[\begin{array}{llll}
1 & 0 & 0 & 1 \\
1 & 1 & 0 & 1 \\
0 & 0 & 0 & 0 \\
0 & 0 & 0 & 1
\end{array}\right] \text {. }
$$

Lemma 5 For $2 \leq i \leq d-1$ and $1 \leq k \leq(d-1)(n-1)$, the restriction of $\beta_{i}$,

$$
\beta_{i}:\left\{P \in \mathcal{C}(d, n): \Theta_{M_{i-1, i}}(P)=k\right\} \rightarrow\left\{P \in \mathcal{C}(d, n): \Theta_{M_{i, 1}}(P)=k\right\}
$$

is a bijection with $\Theta_{M_{i-1, i}}(P)=\Theta_{M_{i 1}}\left(\beta_{i}(P)\right)$. Hence $\Theta_{M_{i-1, i}}$ and $\Theta_{M_{i, 1}}$ are equidistributed.

Proof. We note that $\beta_{i}(P) \in \mathcal{C}(d, n)$ since the condition $x_{1} \leq x_{2} \leq \cdots \leq x_{d}$ holds for any point $\left(x_{1}, x_{2}, \ldots, x_{d}\right)$ on $P$ during the exchanging. The action of $\beta_{i}$ leaves the number of $X_{j} X_{j}$, for $j \neq i$, fixed and exchanges the total number of $X_{h} X_{j}$ pairs, for all $h, j$ where $h<i<j$, with an equal total number of $X_{j} X_{h}$ pairs. Hence, $\Theta_{M_{i-1, i}}(P)=$ $\Theta_{T_{i} M_{i-1, i}}\left(\beta_{i}(P)\right)$, which equals $\Theta_{M_{i, 1}}\left(\beta_{i}(P)\right)$. 
Proof of Proposition 2: This uses Lemmas 3, 4, and 5. Specifically, the identities

$$
\begin{aligned}
\operatorname{des}(P) & =(d-1) n-\Theta_{M_{11}}(P) \\
\Theta_{M_{11}}(P) & =\Theta_{M_{12}}(P) \\
\Theta_{M_{12}}(P) & =\Theta_{M_{21}}\left(\beta_{2}(P)\right) \\
\Theta_{M_{21}}\left(\beta_{2}(P)\right)= & \Theta_{M_{23}}\left(\beta_{2}(P)\right)+1 \\
\Theta_{M_{23}}\left(\beta_{2}(P)\right)= & \Theta_{M_{31}}\left(\beta_{3}\left(\beta_{2}(P)\right)\right) \\
\Theta_{M_{31}}\left(\beta_{3}\left(\beta_{2}(P)\right)\right)= & \Theta_{M_{34}}\left(\beta_{3}\left(\beta_{2}(P)\right)\right)+1 \\
\vdots & \vdots \\
\Theta_{M_{d-2, d-1}\left(\beta_{d-2}\left(\cdots\left(\beta_{2}(P)\right)\right)\right)}= & \Theta_{M_{d-1,1}}\left(\beta_{d-1}\left(\beta_{d-2}\left(\cdots\left(\beta_{2}(P)\right)\right)\right)\right) \\
\Theta_{M_{d-1,1}}\left(\beta_{d-1}\left(\beta_{d-2}\left(\cdots\left(\beta_{2}(P)\right)\right)\right)\right)= & \Theta_{M_{d-1, d}}\left(\beta_{d-1}\left(\beta_{d-2}\left(\cdots\left(\beta_{2}(P)\right)\right)\right)+1\right. \\
\Theta_{M_{d-1, d}}\left(\beta_{d-1}\left(\beta_{d-2}\left(\cdots\left(\beta_{2}(P)\right)\right)\right)\right)= & (d-1) n-1-\operatorname{asc}\left(\beta_{d-1}\left(\beta_{d-2}\left(\cdots\left(\beta_{2}(P)\right)\right)\right)\right.
\end{aligned}
$$

yield the bijective result

$$
\operatorname{des}(P)=\operatorname{asc}\left(\beta_{d-1}\left(\beta_{d-2}\left(\cdots\left(\beta_{2}(P)\right)\right)\right)+d-1 .\right.
$$

Proposition 5 For any $d \geq 2$, suppose that $\Theta_{1}$ is distributed by a reciprocal polynomial of degree $(d-1)(n-1)$ on $\mathcal{C}(d, n)$. If $\Theta_{1}(P)+\Theta_{2}(P)=(d-1)(n-1)$ for all $P \in \mathcal{C}(d, n)$, then they are equidistributed. Specifically, for each statistic $\Theta$ of $\S 3.1$ there is some constant $c$ such that $|\{P \in \mathcal{C}(d, n): \Theta(P)=k+c\}|=N(d, n, k)$.

Proof.

$$
\sum_{P \in \mathcal{C}(d, n)} t^{\Theta_{2}(P)}=\sum_{P \in \mathcal{C}(d, n)} t^{(d-1)(n-1)-\Theta_{1}(P)}=\sum_{P \in \mathcal{C}(d, n)} t^{\Theta_{1}(P)} .
$$

This identity with Lemma 1 implies that each statistic considered, shifted as necessary, has the $d$-Narayana distribution.

Remark on the collection of statistics encoded by 0-1 matrices: For each dimension $d$, how many statistics are there that have a (shifted) $d$-Narayana distribution and can be encoded by a 0-1 matrix? For $d=2,3,4,5$, resp., computations for small values of $n$ show that there are at most 4,24,88,470, resp., 0-1 matrices $M$ such that the statistic $\Theta_{M}$ is distributed by $N(d, n, k-c)$ for some $c$. We say that two statistics, $\Theta$ and $\Theta^{\prime}$ are equivalent if either $\Theta+\Theta^{\prime}$ is constant on $\mathcal{C}(d, n)$ for $n \geq 1$ or $\Theta-\Theta^{\prime}$ is constant on $\mathcal{C}(d, n)$ for $n \geq 1$. For any 0-1 matrix $M$ having exactly one zero row and exactly one zero column, Lemma 1 shows that $\Theta_{M}$ and $\Theta_{H M}$ are equivalent; likewise, $\Theta_{M}$ and $\Theta_{V M}$ are equivalent. For $d=2,3,4,5$, resp., simple computations show that there are $1,4,11,47$, resp., equivalence classes, all of size $2 d$. Each class is generated by $M, H M$, 
$V(H M), H(V(H M)) \ldots$ Using the restricted transposes $T_{i}$ and the bijections $\beta_{i}$, we are able to show that, for any $d$, there are at least $2^{d-2}$ equivalence classes of these matrixencoded statistics which indeed have the (shifted) Narayana distribution. For example for $d=4$, the four classes have as representatives the statistics encoded by $M_{D}, M_{21}, M_{A}$, and $T_{2} M_{32}$ defined in Figure 2. In general, we have yet to show systematically that the other matrix-encoded statistics have the Narayana distribution.

Let $\tau$ be any permutation of $[d]$ and define the statistic $\operatorname{asc}_{\tau}$ on $\mathcal{C}(d, n)$ so

$$
\operatorname{asc}_{\tau}(P):=\left|\left\{i: p_{i} p_{i+1}=X_{\tau_{j}} X_{\tau_{\ell}}, j<\ell\right\}\right| .
$$

It is easily checked that $\operatorname{asc}_{\tau}$ corresponds to a 0-1 matrix $M$ satisfying $\operatorname{asc}_{\tau}=\Theta_{M}$. With $\operatorname{DES}(\tau)$ denoting the number of descents on $\tau$, we conjecture that Proposition 2 generalizes to

$$
\left|\left\{P \in \mathcal{C}(d, n): \operatorname{asc}_{\tau}(P)=k+\operatorname{DES}\left(\tau^{-1}\right)\right\}\right|=N(d, n, k) .
$$

\subsection{High descents}

On any path $P=p_{1} p_{2} \ldots p_{d n} \in \mathcal{C}(d, n)$, the intermediate vertex of the step pair $p_{i} p_{i+1}$ has the coordinates of the final point of the step $p_{i}$. For $d=2$, on any path a high peak is any $Y X$ step pair whose intermediate vertex has coordinates $(x, y)$ satisfying $y-x>1$. Deutsch [4] found that the number of high peaks has the Narayana distribution on $\mathcal{C}(2, n)$.

Now, for arbitrary $d \geq 2$, on any path $P=p_{1} p_{2} \ldots p_{d n} \in \mathcal{C}(d, n)$, call any step pair $p_{i} p_{i+1}$ a high descent if $p_{i} p_{i+1}=X_{j} X_{\ell}$ for $j>\ell$ and its intermediate vertex $\left(x_{1}, x_{2}, \ldots, x_{d}\right)$ satisfies $x_{j}-x_{\ell}>1$. Let hdes $(P)$ denote the number of high descents on the path $P$. By comparing the values of all statistics, which have been considered, on the paths of $\mathcal{C}(3,3)$, one can see that the statistic hdes is not equivalent to any of the others.

Counting with respect to high descents is much closer to counting with respect to ascents than with respect to descents. Specifically, we simply modify the labeling assigned to $\mathbf{d} \times \mathbf{n}$ in Section 2 to

$$
\omega: \mathbf{d} \times \mathbf{n} \rightarrow[d n]: \omega(i, j)=i+d(j-1) .
$$

Again, we also label each $(i, j) \in \mathbf{d} \times \mathbf{n}$ by the step $X_{d-i+1}$. By checking how a descent can occur in any permutation belonging to $\mathcal{L}(\mathbf{d} \times \mathbf{n}, \omega)$, we see that there is a simple bijection mapping each $\tau \in \mathcal{L}(\mathbf{d} \times \mathbf{n}, \omega)$ with $\operatorname{DES}(\tau)=k$ to a path $P \in N(d, n, k)$ with $\operatorname{hdes}(P)=k$. For example, with $d=3$ and $n=2$, see Figure 4 . As a consequence of Propositions 3, we have the following result.

Proposition 6 For any $d \geq 2$ and $0 \leq k \leq(d-1)(n-1)$,

$$
\mid\{P \in \mathcal{C}(d, n): P \text { has } k \text { high descents }\} \mid=N(d, n, k) .
$$

Remark: Brändén [2] has introduced other 2-dimensional Narayana statistics by specifying other labeling linear extension $\omega: \mathbf{2} \times \mathbf{n} \rightarrow[2 n]$. One can do the same for $\mathbf{d} \times \mathbf{n}$, $d>2$. 

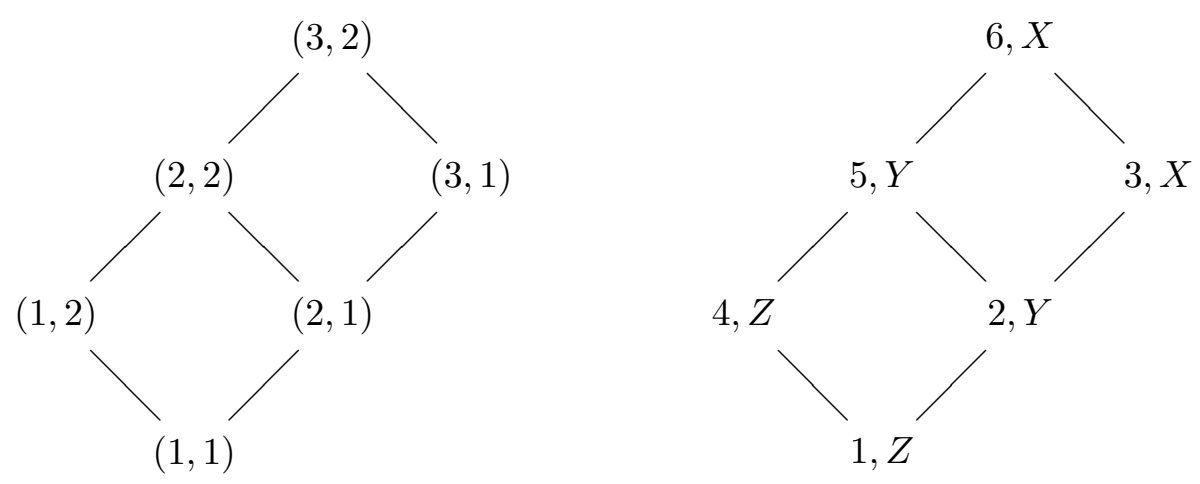

Figure 4: The poset $\mathbf{d} \times \mathbf{n}=\mathbf{3} \times \mathbf{2}$ and its labeled version.

\section{Recurrences}

For $d=2$ and $n \geq 3$, we have the following recurrence for the Narayana polynomial, with bijective proofs appearing in [22, 24]:

$$
(n+1) N_{2, n}(t)=(2 n-1)(1+t) N_{2, n-1}(t)-(n-2)(1-t)^{2} N_{2, n-2}(t) .
$$

For $t=2$, (9) becomes a recurrence for Schröder numbers which was considered bijectively by Foata and Zeilberger [6]. For $d>2$, we are now interested in finding the recurrences for the $d$-Narayana polynomial and for the $d$-Narayana distribution. Perhaps they are amenable to bijective interpretation.

To find and prove a recurrence for the 3-Narayana polynomial, we will apply the algorithm MultiSum of Wegschaider [26] which advances Wilf and Zeilberger's [27] method, a generalization of Sister Celine's method, for handling multiple summations. We will follow the procedures documented in [26]. Here we are not giving computer programs; we are simply giving instructions for the use of Wegschaider's algorithm. We have not recorded the Mathematica outputs in the following as they are easily reproducible and some are quite long. Currently the Mathematica algorithm MultiSum is being enhanced by, and is available from, Axel Riese [15].

Proposition 7 For $n \geq 4$, the 3-Narayana polynomial satisfies

$$
\begin{aligned}
& (3 n-4)(n+2)(n+1)^{2} N_{3, n}(t)= \\
& \quad(3 n-2)(n+1)\left(4\left(1+t+t^{2}\right)-5\left(1+7 t+t^{2}\right) n+3\left(1+7 t+t^{2}\right) n^{2}\right) N_{3, n-1}(t) \\
& \quad-(n-2)\left(-12+29 n-30 n^{2}+9 n^{3}\right)(1-t)^{4} N_{3, n-2}(t) \\
& \quad+(3 n-1)(n-2)(n-3)(n-4)(1-t)^{6} N_{3, n-3}(t) .
\end{aligned}
$$

Proof. Once MultiSum is installed in a Mathematica session, we find and prove this recurrence by executing the following commands, which returns a certificate recurrence, which when summed and then simplified, yields the above recurrence. 


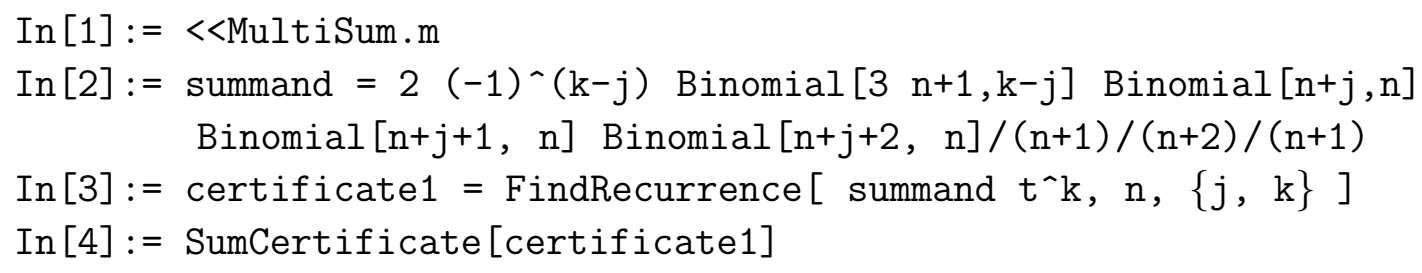

(These commands returned the certificate recurrence, certificate1, of several pages in length in approximately 10 minutes on a 667MHz Pentium III cpu. For the Narayana polynomials for $d=2$ and for $d=4$, similar commands returned certificate recurrences in approximately 4 seconds and 24 hours, respectively.)

Corollary 2 For $n \geq 1, N_{3, n}(t)$ is a reciprocal polynomial.

Proof. This follows from the proposition by induction. This is also a special case of Corollary 1.

Proposition 8 A formula for the 3-dimensional Catalan numbers is

$$
N_{3, n}(1)=\frac{2(3 n) !}{n !(n+1) !(n+2) !}
$$

Proof. First we find a recurrence for the 3-Narayana polynomial evaluated at $t=1$.

In [5]:= certificate 2 = FindRecurrence [ summand, $\mathrm{n},\{\mathrm{j}, \mathrm{k}\}]$

It is then not difficult to guess a simplified formula for $N_{3, n}(1)$ based on the following output:

$$
\begin{aligned}
& \operatorname{In}[6]:=\operatorname{rec}=\text { SumCertificate }[\text { certificate2] }[[1]] \\
& \text { Out }[6]:=-3(-2+3 \mathrm{n})(-1+3 \mathrm{n}) \operatorname{SUM}[-1+\mathrm{n}]+(1+\mathrm{n})(2+\mathrm{n}) \operatorname{SUM}[\mathrm{n}]==0
\end{aligned}
$$

We use

$$
\begin{aligned}
& \operatorname{In}[7]:=\text { guess }=2(3 \mathrm{n}) ! / \mathrm{n} ! /(\mathrm{n}+1) ! /(\mathrm{n}+2) ! \\
& \operatorname{In}[8]:=\text { CheckRecurrence }[\mathrm{rec}, \text { guess }]
\end{aligned}
$$

in order to check that our guess satisfies the recurrence. We complete the proof by checking that the initial value for the guess is correct. (These commands returned a certificate recurrence in approximately 6 minutes; for the case $d=4$, similar commands returned a certificate recurrence in approximately 95 minutes.)

We now consider finding directly a recurrence for the numbers $N(d, n, k)$. We first seek the recurrence in the case of $d=3$. Using MultiSum, we execute the following, obtaining a certificate (in just a few seconds) whose summation simplifies to recurrence (10) for $d=3$ :

$$
\begin{aligned}
& \text { In }[9]:=\text { certificate3 }=\text { FindRecurrence }[\text { summand, }\{n, k\}, j] \\
& \text { In }[10]:=\text { SumCertificate }[\text { certificate }]
\end{aligned}
$$

Then, after using MultiSum similarly to find the recurrence for $d=4$ and $d=5$, we conjecture the formulation given in (10) for any $d$. 
Proposition 9 For $d \geq 2$ and $0 \leq k \leq(d-1)(n-1)$, the numbers $N(d, n, k)$ satisfy

$$
\left(\begin{array}{c}
n+d-1 \\
d
\end{array}\right) N(d, n, k)=\sum_{h=0}^{d}\left(\begin{array}{c}
(d-1)(n-1)-k+h \\
h
\end{array}\right)\left(\begin{array}{c}
n+k+d-h-1 \\
d-h
\end{array}\right) N(d, n-1, k-h)
$$

Proof. To prove that this recurrence is indeed true for any $d$, we first substitute the right side of (3) appropriately for $N(d, n, k)$ and $N(d, n-1, k-h)$ in (10). Then we observe that the following is sufficient for (10) (i.e., only the terms indexed by $j$, and not the full sum with respective to $j$, needs to be used for the proof): for $0 \leq j \leq k$,

$$
\begin{gathered}
\left(\begin{array}{c}
n+d-1 \\
d
\end{array}\right)(-1)^{k-j}\left(\begin{array}{c}
d n+1 \\
k-j
\end{array}\right) \prod_{i=0}^{d-1}\left(\begin{array}{c}
n+i+j \\
n
\end{array}\right)\left(\begin{array}{c}
n+i \\
n
\end{array}\right)^{-1}= \\
\sum_{h=0}^{d}\left(\begin{array}{c}
(d-1)(n-1)-k+h \\
h
\end{array}\right)\left(\begin{array}{c}
n+k+d-h-1 \\
d-h
\end{array}\right)(-1)^{k-h-j}\left(\begin{array}{c}
d n-d+1 \\
k-j-h
\end{array}\right) \times \\
\prod_{i=0}^{d-1}\left(\begin{array}{c}
n-1+i+j \\
n-1
\end{array}\right)\left(\begin{array}{c}
n-1+i \\
n-1
\end{array}\right)^{-1} .
\end{gathered}
$$

It is routine to show that this is equivalent to

$$
\begin{gathered}
\left(\begin{array}{c}
d n+1 \\
k-j
\end{array}\right)\left(\begin{array}{c}
n+j+d-1 \\
d
\end{array}\right)= \\
\sum_{h=0}^{d}(-1)^{h}\left(\begin{array}{c}
d n-n-d+1-k+h \\
h
\end{array}\right)\left(\begin{array}{c}
n+k+d-h-1 \\
d-h
\end{array}\right)\left(\begin{array}{c}
d n-d+1 \\
k-j-h
\end{array}\right)
\end{gathered}
$$

which is a special form of the well-known Pfaff-Saalschütz identity (which can be quickly proven using MultiSum).

\section{5 d-Schröder numbers and a " $2^{n-1}$ result"}

During the past decade the Schröder numbers have received considerable attention, for instance in $[1,6,14,16,21,19]$. For arbitrary $d \geq 2$, we generalize the definitions of the small and large Schröder numbers (as seen in [24]): Let the small and large d-Schröder numbers, respectively, be the sequences $\left(N_{d, n}(2)\right)_{n \geq 1}$ and $\left(2^{d-1} N_{d, n}(2)\right)_{n \geq 1}$, respectively. In each sequence we will set the term for $n=0$ to be 1 . For $d=3$ we have

$$
\begin{gathered}
\left(N_{3, n}(2)\right)_{n \geq 0}=1,1,11,197,4593,126289,3888343,130016393,4629617873, \ldots \\
\left(4 N_{3, n}(2)\right)_{n \geq 1}=4,44,788,18372,505156,15553372,520065572,18518471492, \ldots
\end{gathered}
$$

Consider $d$-dimensional lattice paths using nonzero steps of the form $\left(\xi_{1}, \xi_{2}, \ldots, \xi_{d}\right)$ where $\xi_{i} \in\{0,1\}$ for $1 \leq i \leq d$. Let $\mathcal{D}(n)$ denote the set of paths running from $(0,0, \ldots, 0)$ 
to $(n, n, \ldots, n)$, using these steps, and lying in the region $\left\{\left(x_{1}, x_{2}, \ldots, x_{d}\right): 0 \leq x_{1} \leq x_{2} \leq\right.$ $\left.\ldots \leq x_{d}\right\}$. For $d=2$, such paths are known as (large) Schröder paths, and it is well known that $|\mathcal{D}(n)|=2 N_{2, n}(2)$ for $n \geq 1$.

Proposition 10 For any $d \geq 2$ and $n \geq 1,|\mathcal{D}(n)|=2^{d-1} N_{d, n}(2)$.

Proof. This proof for $d=3$ can easily be generalized. Let $\mathcal{C}^{\prime}(n)$ denote the set of replicated paths formed from the paths of $\mathcal{C}(3, n)$ by independently coloring with $B$ or $R$ the intermediate vertices of $Y X, Z X$, and $Z Y$, i.e., intermediate vertices of descents. Color all other vertices with $R$. Define

$$
\mu: \mathcal{D}(n) \longrightarrow \mathcal{C}^{\prime}(n)
$$

to be the bijection that first sequentially applies the following replacement rules to the diagonal steps of each path:

$$
\begin{aligned}
& (1,1,0) \longrightarrow Y B X \\
& (1,0,1) \longrightarrow Z B X \\
& (0,1,1) \longrightarrow Z B Y \\
& (1,1,1) \longrightarrow Z B Y B X,
\end{aligned}
$$

and then leaves the steps $(1,0,0),(0,1,0)$, and $(0,0,1)$ unaltered, and finally assigns the color $R$ to all non- $B$ vertices on the resulting path. Since $|\mathcal{D}(n)|=\left|\mathcal{C}^{\prime}(n)\right|=$ $\sum_{P \in \mathcal{C}(3, n)} 2^{\operatorname{des}(P)}=2^{2} N_{3, n}(2)$ the result follows.

Next we relate the $d$-Schröder numbers to constrained paths using steps of arbitrary length. Consider those $d$-dimensional lattice paths that use the nonzero steps of the form $\left(\xi_{1}, \xi_{2}, \ldots, \xi_{d}\right)$ where $\xi_{i}$ is a nonnegative integer. Let $\mathcal{S}(n)$ denote the set of paths running from $(0,0, \ldots, 0)$ to $(n, n, \ldots, n)$, using these steps, and lying in the region $\left\{\left(x_{1}, x_{2}, \ldots, x_{d}\right): 0 \leq x_{1} \leq x_{2} \leq \ldots \leq x_{d}\right\}$.

Lemma 6 For $d=3$ and the notation for $\Theta_{M}$ of the previous section, let $M^{\star}=\left[\begin{array}{lll}1 & 0 & 0 \\ 1 & 1 & 0 \\ 1 & 1 & 1\end{array}\right]$.

$$
|\mathcal{S}(n)|=\sum_{P \in \mathcal{C}(3, n)} 2^{\Theta_{M^{\star}}(P)} .
$$

This result generalizes to any $d \geq 2$ where matrix $M^{\star}$ is the $d$ by $d$ matrix defined so that $\left(M^{\star}\right)_{j \ell}=1$ if $j \geq \ell$, and $=0$ if otherwise.

Proof. This proof for $d=3$ can easily be generalized. Let $\mathcal{C}^{\prime \prime}(n)$ denote the set of replicated paths formed from the paths of $\mathcal{C}(3, n)$ by independently coloring with $B$ or $R$ the intermediate vertices of $X X, Y X, Y Y, Z X, Z Y$, and $Z Z$. Color all other vertices with $R$. We define

$$
\nu: \mathcal{S}(n) \longrightarrow \mathcal{C}^{\prime \prime}(n)
$$


to be the bijection that first sequentially applies the following replacement rules to the steps of each path: for $x>0, y>0$, and $z>0$,

$$
\begin{aligned}
& (x, 0,0) \longrightarrow X(B X)^{x-1} \\
& (0, y, 0) \longrightarrow Y(B Y)^{y-1} \\
& (0,0, z) \longrightarrow Z(B Z)^{z-1} \\
& (x, y, 0) \longrightarrow Y(B Y)^{y-1}(B X)^{x} \\
& (x, 0, z) \longrightarrow Z(B Z)^{z-1}(B X)^{x} \\
& (0, y, z) \longrightarrow Z(B Z)^{z-1}(B Y)^{y} \\
& (x, y, z) \longrightarrow Z(B Z)^{z-1}(B Y)^{y}(B X)^{x}
\end{aligned}
$$

and then assigns color $R$ to all non- $B$ vertices on the resulting path. Here the exponents indicate multiple factors in a concatenation; the color $B$ marks intermediate vertices. Since $|\mathcal{S}(n)|=\left|\mathcal{C}^{\prime \prime}(n)\right|=\sum_{P \in \mathcal{C}(3, n)} 2^{\Theta_{M^{\star}}(P)}$ the result follows.

Proposition 11 For any $d \geq 2$ and $n \geq 1,|\mathcal{S}(n)|=2^{d+n-2} N_{d, n}(2)$.

Proof. This proof for $d=3$ can easily be generalized. Since $M^{\star}+M_{A}=\left[\begin{array}{lll}1 & 1 & 1 \\ 1 & 1 & 1 \\ 1 & 1 & 1\end{array}\right]$, $\Theta_{M^{\star}}(P)+\operatorname{asc}(P)=3 n-1$. This fact and Corollary 1 show

$$
\sum_{P \in \mathcal{C}(3, n)} 2^{\Theta_{M^{\star}}(P)}=2^{n+1} \sum_{P \in \mathcal{C}(3, n)} 2^{2 n-2-\operatorname{asc}(P)}=2^{n+1} \sum_{P \in \mathcal{C}(3, n)} 2^{\operatorname{asc}(P)} .
$$

Using the Lemma 6 completes the proof.

Corollary 3 For any $d \geq 2$ and $n \geq 1,|\mathcal{S}(n)|=2^{n-1}|\mathcal{D}(n)|$.

Proof. This $2^{n-1}$ result is a consequence of Propositions 10 and 11.

\section{Remarks:}

5.1: We observe that $\mathcal{D}(n)$ is counted using the statistic des while $\mathcal{S}(n)$ is counted using the statistic asc together with the reciprocity of the $d$-Narayana polynomial.

5.2: The "classic $2^{n-1}$ result" is for $d=1$ : one can easily show $|\mathcal{S}(n)|=2^{n-1}|\mathcal{D}(n)|=$ $2^{n-1}$ (See [11, art. 123].) Our interest in such results, which relate paths using "super steps" (perhaps diagonal) to those using "short steps" (perhaps diagonal), originated from Stanley's exercise [21, ex. 6.16]. For $d=2$ and $n \geq 1$, paper [23] gives a bijection showing that $|\mathcal{S}(n)|=2^{n-1}|\mathcal{D}(n)|=2^{n} N_{2, n}(2)$. Duchi and Sulanke [5] give a bijective proof indicating that for any $d,|\mathcal{S}(n)|=2^{n-1}|\mathcal{D}(n)|$ is true when the constraint $0 \leq x_{1} \leq$ $x_{2} \leq \ldots \leq x_{d}$ is absent. Remarkably, the formula of the " $2^{n-1}$ result" is independent of $d$.

5.3: Our encoding of the paths of $\mathcal{D}(n)$ in the proof of Proposition 10 and paths of $\mathcal{S}(n)$ in the proof of Lemma 6 in terms of paths of $\mathcal{C}(3, n)$ with colored vertices is consistent with the encoding of such steps by MacMahon [11, sect. IV]. 
Acknowledgments: The author is grateful to Axel Riese for his support in using the algorithm MulTiSum. He thanks Herbert Wilf for his productive suggestions pertaining to Section 4. He is appreciative of the local computer support given by Martin Lukes and Angus McDonald. He thanks the referee for several positive suggestions including a comment which led to the conjecture at the end of the remarks of $\S 3.1$.

\section{References}

[1] J. Bonin, L. Shapiro, and R. Simion, Some $q$-analogues of the Schrder numbers arising from combinatorial statistics on lattice paths. J. Statist. Plann. Inference 34 (1993), no. $1,35-55$.

[2] P. Brändén, $q$-Narayana numbers and the flag $h$-vector of $J(\mathbf{2} \times \mathbf{n})$, Discrete Math. 281 (2004), no. 1-3, 67-81.

[3] D.M. Bressoud Proofs and Confirmations, Cambridge Univ. Press, 1999.

[4] E. Deutsch, An involution on Dyck paths and its consequences. Discrete Math. 204 (1999), no. 1-3, 163-166.

[5] E. Duchi and R.A. Sulanke, The $2^{n-1}$ factor for multi-dimensional lattice paths with diagonal steps, Sem. Lothar. Combin, B51d (2003), 16 pp.

[6] D. Foata and D. Zeilberger, A classic proof of a recurrence for a very classical sequence. J. Combin. Theory Ser. A 80 (1997), no. 2, 380-384.

[7] I.M. Gessel and X.G. Viennot, Binomial determinants, paths, and hook length formulae, Adv. Math., 58 (1985) 300-321.

[8] G. Kreweras, Sur une extension du problème dit "de Simon Newcomb", C. R. Acad. Sci. Paris, Ser. A-B 2631966 A43-A45.

[9] G. Kreweras and H. Niederhausen, Solution of an enumerative problem connected with lattice paths. European J. Combin. 2 (1981), no. 1, 55-60.

[10] P.A. MacMahon, Memoir on the theory of the partitions on numbers - Part V, Phil. Trans., (1912), 75-110. This appears in [12].

[11] P.A. MacMahon, Combinatory analysis, 1915-16, Two volumes (bound as one), Chelsea Pub. Co., 1960.

[12] P.A. MacMahon, Collected papers. Vol. I. Combinatorics, Edited by G.E. Andrews, MIT Press, 1978. 
[13] T.V. Narayana, Sur les treillis formés par les partitions d'un entier et leurs applications à la théorie des probabilités, C. R. Acad. Sci. Paris, 240 (1955) 1188-1189.

[14] E. Pergola and R.A. Sulanke, Schröder triangles, paths, and parallelogram polyominoes. J. Integer Seq. 1 (1998), Article 98.1.7

[15] A. Riese Research Institute for Symbolic Computation, J. Kepler University Linz, http://www.risc.uni-linz.ac .at/research/combinat/risc/software/

[16] L. Shapiro and R.A. Sulanke, Bijections for the Schrder numbers. Math. Mag. 73 (2000), no. 5, 369-376.

[17] N.J.A. Sloane, On-line Encyclopedia of Integer Sequences, http://www.research.att.com/ njas/sequences/index.html

[18] R.P. Stanley, Ordered structures and partitions. Memoirs of the Am. Math. Soc., No. 119. Am. Math. Soc, 1972

[19] R.P. Stanley, Hipparchus, Plutarch, Schröder, and Hough. Amer. Math. Monthly 104 (1997), no. 4, 344-350.

[20] R.P. Stanley, Enumerative combinatorics. Vol. 1, Cambridge Univ. Press, 1997.

[21] R.P. Stanley, Enumerative combinatorics. Vol. 2, Cambridge Univ. Press, 1999.

[22] R.A. Sulanke, Bijective recurrences for Motzkin paths, Adv. in Appl. Math. 27 (2001), no. 2-3, 627-640.

[23] R.A. Sulanke, Counting lattice paths by Narayana polynomials, Electron. J. Combin. 7 (2000), no. 1, paper 40, 9 pp.

[24] R.A. Sulanke, The Narayana distribution, J. Statist. Plann. Inference 101 (2002), no. 1-2, 311-326.

[25] R.A. Sulanke, Three dimensional Narayana and Schröder numbers, preprint 2003, to appear in Theoret. Comput. Sci.

[26] K. Wegschaider, Computer Generated Proofs of Binomial Multi-Sum Identities, Diploma Thesis, RISC, J. Kepler University, Linz, May 1997. http://www.risc.uni-linz.ac.at/research/combinat/risc/software/

[27] H. S. Wilf and D. Zeilberger, An algorithmic proof theory for hypergeometric (ordinary and " $q$ ") multisum/integral identities. Invent. Math. 108 (1992), no. 3, 575-633.

[28] D. Zeilberger, André's reflection proof generalized to the many candidate ballot problem. Discrete Math. 44 (1983) 325-326. 